\title{
Combating Fibre Nonlinearity Using Dual-Order Raman Amplification and OPC
}

\author{
Mohammad Al-Khateeb, Mingming Tan, Tingting Zhang, and Andrew D. Ellis
}

\begin{abstract}
We experimentally demonstrate, for the first time, a significant 34dB nonlinear product power compensation in a midlink optical phase conjugation (OPC) system using an optimised dual-order distributed Raman amplification (DRA) technique. The dual-order backward (BW)-pumping scheme shows a record signal power symmetry of $97 \%$ over $50.4 \mathrm{~km}$ single mode fibre (SMF) spans. We also demonstrate that the required accuracy for span-to-span power alignment is within $\pm 1 \mathrm{~dB}$ in order to maintain $20 \mathrm{~dB}$ nonlinear product compensation. For a $256 \mathrm{~Gb} / \mathrm{s}$ dualpolarisation-16QAM transmission over $100.8 \mathrm{~km}(2 \times 50.4 \mathrm{~km}) \mathrm{SMF}$ with mid-link OPC, the proposed Raman amplification scheme enables the OPC to improve the nonlinear threshold by $\sim 7 \mathrm{~dB}$ and the optimum signal launch power by $\sim 5 \mathrm{~dB}$, compared to the system without OPC.
\end{abstract}

Index Terms-Optical amplifiers, optical fibre communication, phase conjugation, nonlinear optics.

\section{INTRODUCTION}

$\mathrm{F}$ ibre nonlinear impairments have been a major issue to restrain the rapidly increasing data capacity demand in optical fibre communications. Several techniques including digital back propagation (DBP), phase conjugated twin-wave, and mid-link optical phase conjugation (OPC), have been investigated to combat such nonlinear impairments [1-3]. OPC has been proven to be a promising all-optical signal processing technique to compensate both linear (mainly dispersive) and nonlinear impairments simultaneously for multiple Wavelength Division Multiplexed (WDM) channels [4-7]. However, the compensation efficiency in a mid-link OPC system is highly dependent on the symmetry of the signal power profile along the fibre (in reference to the middle of the link), which means that full nonlinearity compensation can only be achieved when the signal power is precisely matched on either side of the midlink OPC [3]. Suitable signal power profile symmetry can be enabled by distributed Raman amplification (DRA), instead of conventional lumped amplification [8]. When deploying firstorder DRA for a given span length $[9,10]$, the signal power profile has limited symmetry and can be only improved by shortening the span length (Raman pump spacing). Experimental results in [9] demonstrate that using backwardpumped first-order DRA $(2 \times 50 \mathrm{~km})$ in a mid-link OPC system can achieve a nonlinear product compensation efficiency of

Manuscript received xxxx, 2019; revised xxxx, 2019; accepted xxxx, 2019. Date of publication xxxx, 2019; date of current version xxxxx, 2019.

This work was funded by UK EPSRC Programme Grant EP/L000091/1 (PEACE) and EP/S003436/1 (PHOS). We thank Marc Stephens (with Infinera, UK) for useful discussions. We thank Sterlite Technologies for providing the $\sim 27 \mathrm{~dB}$. Deploying higher order Raman pumping can provide the flexibility in optimising the signal power symmetry along the fibre span [10]. On one hand, for example, in [10,11], Raman fibre laser (RFL) based amplification with bidirectional second-order pumping assisted by fibre Bragg grating (FBG) was used to enhance the signal power symmetry. However, such scheme requires significantly high pump power due to the lower Raman gain efficiency of the forward second-order pumping. Although the pump power can be decreased using the first-order pump, severe Random Intensity Noise (RIN) may be imposed onto the signals from the forward propagating pump, which can result in significant system performance degradation for long-haul coherent transmissions. Therefore, the first-order forward pumping has to be avoided unless RIN transfer can be mitigated. On the other hand, dual-order DRA comprises independently controlled first- and second-order pumps. The previous studies on dual-order DRA have been only focused on providing quasi-constant signal power profile, in which the first-order pump power was kept minimum $[11,13]$. Here, in the mid-link OPC system, dual-order backward (BW)-pumping DRA is designed for the best signal power profile symmetry, by optimising the first- and second-order pump power.

In this paper, we expand our recent work on the optimisation of dual-order DRA systems which demonstrate a 97\% signal power profile symmetry that leads to $34 \mathrm{~dB}$ nonlinearity compensation using mid-link OPC over $2 \times 50.4 \mathrm{~km}$ single mode fibre (SMF) link [14]. The dual-order DRA gives an $7 \mathrm{~dB}$ enhancement to the nonlinearity compensation efficiency of the first-order DRA shown in [9]. Furthermore, we find that the nonlinearity compensation efficiency is very sensitive to the misalignment of the signal power launched into each span, with only $+/-0.9 \mathrm{~dB}$ signal launch power misalignment (per span) to maintain $20 \mathrm{~dB}$ of nonlinearity compensation in the dual-order DRA system. Finally, we present an analytical and experimental study of nonlinear performance limits of a modulated signal (32Gbaud, DP-16QAM, 256Gb/s) that propagates through two spans of dual-order DRA optimised at their highest power profile symmetry (97\%). The results show that deploying a mid-link OPC in the system can achieve a significant $7 \mathrm{~dB}$ improvement in the nonlinear threshold and a $5 \mathrm{~dB}$ enhancement of the optimum signal launch power, compared with the system without OPC [15].

transmission fibre, and Finisar for providing a WSS (Corresponding author: Mingming Tan).

Original data for this work is available through Aston Research Explorer (https://doi.org/10.17036/researchdata.aston.ac.uk.00000409). 


$$
\begin{gathered}
P_{F}^{w o / O P C}=\left(\frac{D \gamma}{3}\right)^{2} \frac{\sin ^{2}(N \Delta \beta L / 4)}{\sin ^{2}(\Delta \beta L / 2)}\left|E_{q} E_{r} E_{s}^{*} \sum_{k=1}^{M}\left[\frac{\mathrm{e}^{\left(g_{k}+i \Delta \beta\right) L_{k}}-1}{g_{k}+i \Delta \beta}\right]\left[\prod_{l=1}^{k-1} \mathrm{e}^{\left(g_{l}+i \Delta \beta\right) L_{l}}\right]+E_{q}^{\prime} E_{r}^{\prime} E_{s}^{* \prime} \sum_{k=1}^{M}\left[\frac{\mathrm{e}^{\left(g_{k}+i \Delta \beta\right) L_{k}}-1}{g_{k}+i \Delta \beta}\right]\left[\prod_{l=1}^{k-1} \mathrm{e}^{\left(g_{l}+i \Delta \beta\right) L_{l}}\right]\right|^{2}(1) \\
P_{F}^{w / O P C}=\left(\frac{D \gamma}{3}\right)^{2} \frac{\sin ^{2}(N \Delta \beta L / 4)}{\sin ^{2}(\Delta \beta L / 2)}\left|E_{q} E_{r} E_{s}^{*} e^{(-i \Delta \beta L)} \sum_{k=1}^{M}\left[\frac{\mathrm{e}^{\left(g_{k}+i \Delta \beta\right) L_{k}}-1}{g_{k}+i \Delta \beta}\right]\left[\prod_{l=1}^{k-1} \mathrm{e}^{\left(g_{l}+i \Delta \beta\right) L_{l}}\right]-E_{q}^{* \prime} E_{r}^{* \prime} E_{s}^{\prime} \sum_{k=1}^{M}\left[\frac{\mathrm{e}^{\left(g_{k}-i \Delta \beta\right) L_{k}}-1}{g_{k}-i \Delta \beta}\right]\left[\prod_{l=1}^{k-1} \mathrm{e}^{\left(g_{l}-i \Delta \beta\right) L_{l}}\right]\right|^{2}
\end{gathered}
$$

\section{TheORETICAL EVAluation}

An analytical description of the nonlinear frequency response of an optical transmission system (that deploys DRA) can be written as Eq.(1) [9], and Eq.(2) shows nonlinear frequency response of a DRA system that deploys a mid-link OPC. These analytical equations find the coherent summation of the nonlinear interactions among one (Self-Phase Modulation, SPM), two (including cross-Phase Modulation, XPM) or three waves (Four-Wave Mixing, FWM) along $N$ spans of length $L$ with identical signal power profile. This method subdivides the DRA span into $M$ sections, where each section has a constant logarithmic gain or loss that is calculated from the sampled linear interpolation of the power profile. In both equations, $\mathrm{E}_{\mathrm{q}}, \mathrm{E}_{\mathrm{r}}, \mathrm{E}_{\mathrm{s}}$ represent the amplitude of the mixing optical fields, $D$ represents degeneracy factor, $\gamma$ is the fibre's nonlinear coefficient, $\Delta \beta\left(=-4 \pi 2 \beta_{2}\left[f_{q}-f_{s}\right]\left[f_{r}-f_{s}\right]\right)$ is the phase mismatching coefficient, $L_{k}$ is the $\mathrm{k}^{\text {th }}$ section length, and $g_{k}$ represents exponential gain/loss coefficient for the $\mathrm{k}^{\text {th }}$ section. Equation (1) represents a discretised version of the continuous integral [16] and has been modified to represent the case of different signal powers in each half of the link. Outside the modulus (in Eq.(1) and Eq.(2)) are a constant scaling factor and oscillating quasi-phase matching term. Inside the modulus, we have the coherent summation of the nonlinear optical field generated from each segment in the spans of the first and the second halves of the link. On one hand, without an OPC, any misalignment in signal launch power only results in a variation in the nonlinear product power in the strongly phase matched region (i.e. $\Delta \beta \rightarrow 0$ ) due to the change in power itself. On the other hand, with an OPC there may be a significant variation away from ideal compensation in the strongly phase matched region (i.e. $\Delta \beta \rightarrow 0$ ). Fig.1(a) shows that, for small offsets $( \pm 2 \mathrm{~dB})$, the nonlinear product power varies by only $3 \mathrm{~dB}$ for a system without an OPC while at least $30 \mathrm{~dB}$ for a system with an OPC.

\section{EXPERIMENTAL SETUP}

Figure 1(b) depicts the experimental setup of nonlinear Kerr product measurement which can verify Eqns. (1\&2). The efficiency of nonlinear product (XPM) generation was evaluated by injecting two Continues Wave $(\mathrm{CW})$ lasers $(3 \mathrm{dBm}$ each, $\sim 192.8 \mathrm{THz}$, one fixed, one tuneable) and analysing the output with a $150 \mathrm{MHz}$ resolution optical spectrum analyser (OSA) without and with OPC located in the mid-link. Dualorder backward pumping DRA was used to compensate the $\sim 9.8 \mathrm{~dB}$ loss from the $50.4 \mathrm{~km}$ transmission fibre (Sterlite $\mathrm{OH}$ LITE(E)) of each span. The signal after the first span can be transmitted either without or with OPC, using a lithium-niobite optical switch. The input power to the second span was adjusted by an EDFA. In the OPC path, an additional amplifier was used to partially pre-compensate the insertion loss of the OPC $(\sim 20 \mathrm{~dB})$. The detailed experimental setup of the OPC is shown in Fig. 1(b) (bottom). The configuration supports polarisation insensitive, dual-band, dual-pump signal conjugation, but we used only one signal band (that contains the two CW lasers). The two pump lasers (linewidth $<10 \mathrm{kHz}$, at $1540.7 \mathrm{~nm}$ and $1570.4 \mathrm{~nm}$ ) were counter-dithered (phase modulated) with two tones (at $60 \mathrm{MHz}$ and $600 \mathrm{MHz}$ ) to increase the stimulated Brillouin scattering (SBS) threshold of the highly nonlinear fibre (HNLF) by $8 \mathrm{~dB}$. The phases of the RF tones were selected to minimise the phase modulation transfer from the pumps to the conjugated signals. Polarisation controllers (PC) and polarisation beam splitters (PBS) were used to polarize the two pumps. The two pump lasers were amplified by polarisation maintaining (PM)-EDFA and then filtered by circulators and fibre Bragg gratings (FBGs) at their respective wavelengths. Then, the two orthogonally-polarised pumps were combined using a polarisation beam combiner (PBC), with a 50/50 splitter utilized to generate two copies of the orthogonally polarized

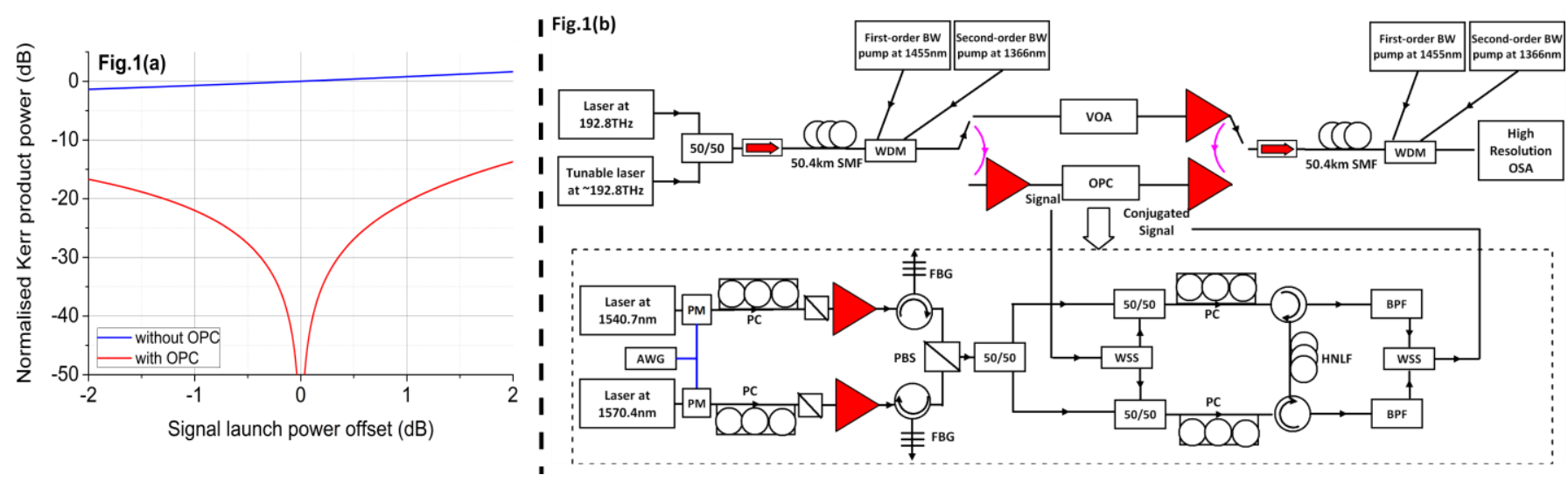

Fig. 1(a). Normalised Kerr product power as a function of the signal launch power offset. Fig. 1(b). Experimental setup of nonlinear product measurement using dual-order DRA in mid-link OPC systems 
pumps. At the signal input of the OPC, a WSS was used to filter out-of-the band ASE noise. The filtered signal and a copy of the orthogonally polarised pumps were combined with a 50/50 coupler. The polarisation controllers were installed on each path to align the pumps with the principal axis of the HNLF and minimise the polarisation walk-off. Thus, the polarisation independence of the conjugation was maintained. The signal combined with the pumps were bi-directionally propagated in $\operatorname{HNLF}\left(\mathrm{L}=100 \mathrm{~m}, \lambda_{0}=1557, \gamma_{0}=28 / \mathrm{W} / \mathrm{km}, \mathrm{S}=0.024 \mathrm{ps} / \mathrm{nm}^{2} / \mathrm{km}\right.$ ) via two circulators, as shown in Fig. 2. The circulators allow the signal, the conjugate, and two pumps to be extracted after passing through the HNLF. Tuneable optical band pass filters (BPFs) and a WSS were used to remove the high-power pumps.

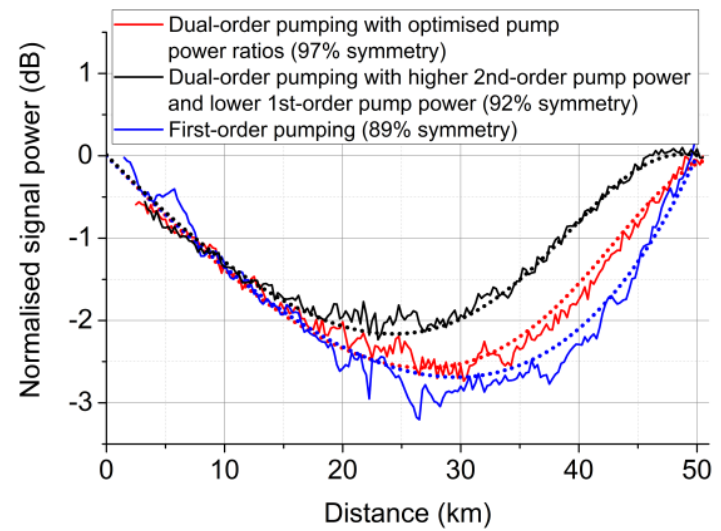

Fig. 2. Simulated and measured signal power profiles using different DRA schemes including dual-order BW-pumping DRA with optimised pump power ratios, dual-order BW-pumping DRA with higher second-order power and lower first-order power, and first-order BW-pumping DRA

Figure 2 shows the simulated and experimentally measured signal power profiles along the fibre using different DRA schemes. Dual-order BW-pumping scheme can significantly improve the symmetry of signal power profile, but it requires the optimisation of the first- and second-order pump power. The first-order BW-pumping scheme provides $89 \%$ signal power symmetry (ref. to [10] for calculation) over $50.4 \mathrm{~km} \mathrm{SMF} \mathrm{(based}$ on the simulation in Fig. 2). The first-order pump power was $\sim 360 \mathrm{~mW}$. To achieve $97 \%$ symmetry, dual-order BW-pumping was precisely optimised. The first- and second-order pump power was $\sim 100 \mathrm{~mW}$ and $\sim 330 \mathrm{~mW}$ respectively, which the total pump power was increased slightly by $\sim 70 \mathrm{~mW}$. The signal power symmetry was degraded using $\sim 33 \mathrm{~mW}$ and $\sim 600 \mathrm{~mW}$ for the first- and second-order pump power, respectively. This asymmetry was mainly due to the signal power gain occurred toward the end of the fibre, as the signal gain was pushed into the middle span using higher second-order pump power [11].

\section{RESULTS AND DISCUSSIONS}

The Kerr product power and the noise floor, without and with $\mathrm{OPC}$, is presented in Fig. 3(a), when varying with the frequency separation between the two CW lasers. The generated nonlinear product power without OPC was up to $\sim-11.5 \mathrm{~dB}$ at strongly phase matched region and oscillated with the frequency separation due to the phase mismatching accumulation $(\Delta \beta$ in Eq. [1]). Using the mid-link OPC, the noise floor power was increased by $\sim 9 \mathrm{~dB}$ because of the insertion loss of the OPC and the additional amplifier. However, the Kerr product power was suppressed (when deploying the OPC) significantly by a factor of $28.5 \mathrm{~dB}$ in the strongly phase matched region (low frequency separation) and by a factor of $\sim 34 \mathrm{~dB}$ when comparing the maximum nonlinear product power peak $(2.5 \mathrm{GHz}$ separation in the case without OPC to $19 \mathrm{GHz}$ separation in the case with $\mathrm{OPC}$ ). This nonlinear product compensation of $\sim 34 \mathrm{~dB}$ (when comparing the nonlinear product power peak) was achieved using the optimised dual-order DRA scheme, an $\sim 8 \mathrm{~dB}$ enhancement compared with first-order DRA over the same span demonstrated in [9]. Fig. 3(b) and 3(c) shows the impact on the nonlinear product power measurement, when the signal power launched into the second span was misaligned from $0.9 \mathrm{~dB}$ lower to $0.9 \mathrm{~dB}$ higher, both theoretically (Fig. 3(b)) and experimentally (Fig. 3(c)). It can be seen that $+/-0.9 \mathrm{~dB}$ misalignment of signal launch power results in the Kerr product power (generated in the OPC-assisted system) increasing by $\sim 12 \mathrm{~dB}$ in the strongly phase matched region, indicating a $\sim 22 \mathrm{~dB}$ degradation for nonlinear product compensation efficiency. However, for the system without OPC, a very small increase $(\sim 1.5 \mathrm{~dB})$ of Kerr product power is observed due to the signal launch power misalignment $(-0.9$ to $0.9 \mathrm{~dB})$ and negligible changes in null position. Identical trends were obtained from theoretical predictions (calculated from Eq.(1) and Eq.(2), in Fig .3(b)), without considering the noise floor.

An inline coherent transmission experiment was conducted by replacing the two $\mathrm{CW}$ lasers shown in Fig. 1(b) with a 256Gb/s DP-16QAM (32GBaud) signal (centred at 194.8THz). After the transmission, the signal or its conjugation (centred at $194.65 \mathrm{THz}$ ) was amplified by a receiver-side EDFA before detected by a polarisation-diverse coherent receiver $(100 \mathrm{GSa} / \mathrm{s}$,
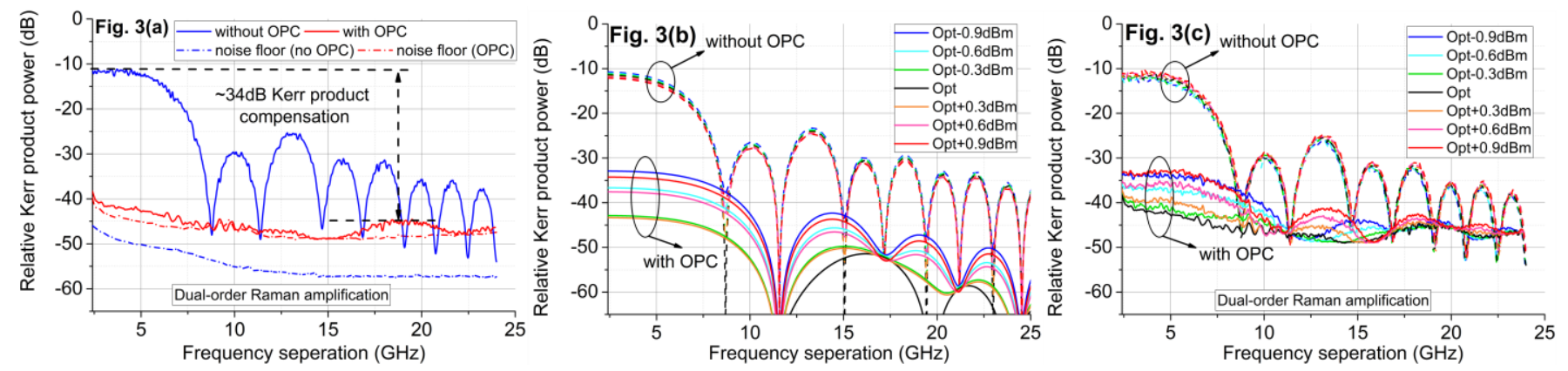

Fig. 3(a). Experimentally measured nonlinear Kerr product power as a function of frequency separation between the two CW lasers; Fig. 3(b) and 3(c). Simulated and experimentally measured nonlinear Kerr product power as a function of frequency separation at different signal launch powers 
33GHz). A commercial digital signal processing software (Tektrnoix OM1106) was used for signal processing with $\mathrm{Q}^{2}$ factors calculated from the bit-error-rate of $500 \mathrm{k}$ bits.

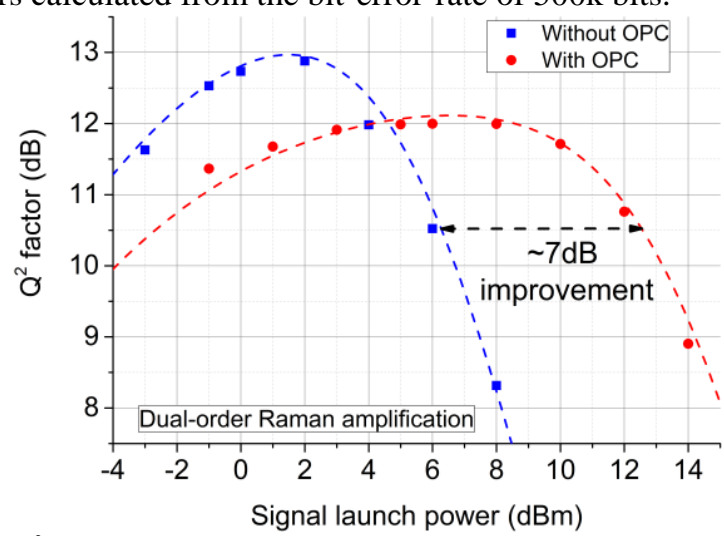

Fig. 4. $Q^{2}$ factors versus signal launch power in inline transmission systems without/with OPC using the optimised dual-order DRA scheme (Dots: experimental results; Dashed line: analytical fit).

Figure 4 shows the experimental and analytically fitted $\mathrm{Q}^{2}$ factors versus signal launch power using the dual-order DRA scheme. The analytical model characterises the SNR of the coherently detected signal considering the transceiver maximum achievable SNR (without OPC and with OPC), which can be presented as follows:

$$
\begin{aligned}
\mathrm{SNR}_{\mathrm{No} O \mathrm{OPC}}=\frac{\mathrm{I}_{\mathrm{s}}}{\frac{\mathrm{I}_{\mathrm{S}}}{\mathrm{SNR}_{\mathrm{TRx} \max }}+\mathrm{I}_{\mathrm{ASE}}+\mathrm{I}_{\mathrm{NL}}} \\
\mathrm{SNR}_{\mathrm{OPC}}=\frac{\mathrm{I}_{\mathrm{S}}}{\frac{\mathrm{I}_{\mathrm{S}}}{\mathrm{SNR}_{\mathrm{TRx}+\mathrm{OPC} \text { max }}}+\mathrm{I}_{\mathrm{ASE}}+\delta \mathrm{I}_{\mathrm{NL}}}
\end{aligned}
$$

where $I_{s}, I_{A S E}$, and $I_{N L}$ are the power spectral densities of signal, linear noise (ASE), and nonlinear noise, respectively. $S N R_{\text {TRx_max }}$ (approximated in our experiment to $\sim 19.8 \mathrm{~dB}$ ) is the maximum SNR with the transceiver in a back-to-back setup, and the $S N R_{T R x+O P C_{-} \max }$ (approximated in our experiment to $\sim 17.5 \mathrm{~dB}$ ) is the maximum SNR with the transceiver and an OPC in a Tx-OPC-Rx configuration. The resulting SNR from Eq. (3) and (4) can be converted to BERs ( $Q^{2}$ factors) at a given modulation format (i.e. 16-QAM) [3]. $\delta$ is the nonlinearity compensation efficiency achieved by the OPC, when a Nyquist modulated signal with a bandwidth $\mathrm{B}_{\mathrm{w}}$ is propagating along the system, which can be defined as follows:

$$
\delta=\frac{\mathrm{I}_{\mathrm{NL}}(\mathrm{wo} / \mathrm{OPC})}{\mathrm{I}_{\mathrm{NL}}(\mathrm{w} \text { OPC })}=\frac{\iint_{-\mathrm{B}_{\mathrm{w}} / 2}^{-\mathrm{B}_{\mathrm{w}} / 2} \mathrm{P}_{\mathrm{F}}^{\mathrm{wo} / O P C} \mathrm{df}_{1} \mathrm{df}_{2}}{\iint_{-\mathrm{B}_{\mathrm{w}} / 2}^{-\mathrm{B}_{\mathrm{w}} / 2} \mathrm{P}_{\mathrm{F}}^{\mathrm{w} / O P C} \mathrm{df}_{1} \mathrm{df}_{2}}
$$

Such calculation can reveal a significant nonlinearity compensation efficiency that can be achieved (assuming 32Gbaud Nyquist signal) by OPC when deployed in dual-order DRA system is $0.04(\sim 14 \mathrm{~dB})$.

In Fig. $4, \sim 7 \mathrm{~dB}$ reduction in the nonlinear threshold and $\sim 5 \mathrm{~dB}$ in optimum launch power have been achieved due to the nearly perfect ( 97\%) signal power symmetry provided by dual-order BW-pumping DRA. Due to the short transmission distance $(100 \mathrm{~km})$, the optimum $\mathrm{Q}^{2}$ factor with OPC was dominated by the transceiver noise in addition to the noise from the OPC and the EDFA, which obscures the benefit of fibre nonlinearity compensation. However, in long-haul transmission, the OPC is expected to significantly improve the system performance, since the transceiver and OPC noise can be negligible compared with the accumulated link noise.

\section{CONCLUSION}

We conduct a detailed study of nonlinearity compensation using an optimised dual-order BW-pumping DRA scheme in a mid-link OPC system. Dual-order DRA scheme achieves 97\% signal power profile symmetry and enables significant $34 \mathrm{~dB}$ nonlinear product compensation when deploying the mid-link OPC. We theoretically and experimentally study the impact of the signal launch power misalignment on the nonlinear product compensation efficiency. The results show that a $0.9 \mathrm{~dB}$ signal power offset degrades the compensation efficiency by $12 \mathrm{~dB}$. Furthermore, we experimentally and analytically demonstrate that the proposed Raman scheme allows $7 \mathrm{~dB}$ improvement in the nonlinear threshold and $5 \mathrm{~dB}$ enhancement of optimum signal launch power with the OPC-assisted $256 \mathrm{~Gb} / \mathrm{s}$ DP16QAM inline transmission system over 100km SMF.

\section{REFERENCES}

[1] T. Xu et al., "Digital nonlinearity compensation in high-capacity optical communication systems considering signal spectral broadening effect," Scientific reports, vol. 7, 12986, 2017.

[2] X. Liu et al., "Phase-conjugated twin waves for communication beyond the Kerr nonlinearity limit," Nature Photonics, vol.7, pp.560-568, 2013.

[3] A. D. Ellis et al., "Performance limits in optical communications due to fiber nonlinearity," Adv. Opt. Photon., vol.9, pp.429-503, 2017.

[4] H. Hu et al., "Fiber nonlinearity mitigation of WDM-PDM QPSK/16QAM signals using fiber-optic parametric amplifiers based multiple optical phase conjugations," Opt. Express, vol. 25, pp. 1618-1628, 2017.

[5] S. Yoshima et al., "Mitigation of Nonlinear Effects on WDM QAM Signals Enabled by Optical Ph ase Conjugation With Efficient Bandwidth Utilization," J. Lightwave Technol., vol. 35, pp. 971-978, 2017.

[6] K. Solis-Trapala et al., "Optimized WDM Transmission Impairment Mitigation by Multiple Phase Conjugations," J. Lightwave Technol., vol. 34, pp. 431-440, 2016.

[7] T. Umkei et al., "Simultaenous nonlinearity mitigation in $92 \times 180 \mathrm{Gbit} / \mathrm{s}$ PDM-16QAM transmission over $3840 \mathrm{~km}$ using PPLN-based guard-bandless optical phase conjugation," Opt. Express, vol. 24, no. 15, pp. 16945$16951,2016$.

[8] M. A. Z. Al-Khateeb et al., "Experimental Verification of Four Wave Mixing in Lumped Optical Transmission Systems that Employ Mid-Link Optical Phase Conjugation," in Conference on Lasers and Electro-Optics (CLEO) 2017, paper. JTh2A.64.

[9] M. A. Z. Al-Khateeb et al., "Analysis of the nonlinear Kerr effects in optical transmission systems that deploy optical phase conjugation," Opt. Express, vol. 26, pp. 3145-3160, 2018.

[10] P. Rosa et al., "Signal power asymmetry optimisation for optical phase conjugation using Raman amplification," Opt. Express, vol. 23, pp. 31772-31778, 2015.

[11] M. Tan et al., "Transmission performance improvement using random DFB fiber laser based Raman amplification and bidirectional secondorder pumping," Opt. Express, vol. 24, no. 3, pp. 2215-2221, 2016.

[12] M. Tan et al., "RIN Mitigation and Transmission Performance Enhancement With Forward Broadband Pump," IEEE Photon. Technol. Lett., vol. 30, no. 3, pp. 254-257, Feb.1, 12018.

[13] J.-C. Bouteiller et al., "Dual-order Raman pump providing improved noise figure and large gain bandwidth," in $O F C 2002$, p. FB3.

[14] M. A. Z. Al-Khateeb et al., "Symmetry Requirements for $34 \mathrm{~dB}$ Nonlinearity Compensation in OPC Systems," in ECOC 2018, p. We3H.3.

[15] M. Morshed et al., "Experimental demonstrations of dual polarization CO-OFDM using mid-span spectral inversion for nonlinearity compensation," Opt. Express, vol. 22, no. 9, pp. 10455-10466, 2014. 
[16] S. Radic et al., "Four-wave mixing in optical links using quasi-distributed optical amplifiers," J. Light. Technol. Vol.19, no. 5, 636-645, 2001. 